\title{
The Enlightment and Reference of Foreign Experience on the Values Education Practice in Chinese Universities
}

\author{
Zhang Wenling \\ Center for Ideological and Political Education, Northeast Normal University, Changchun, P.R.China
}

Email address:

980707796@qq.com

To cite this article:

Zhang Wenling. The Enlightment and Reference of Foreign Experience on the Values Education Practice in Chinese Universities. Science Innovation. Vol. 6, No. 2, 2018, pp. 92-96. doi: 10.11648/j.si.20180602.17

Received: April 19, 2018; Accepted: May 7, 2018; Published: June 22, 2018

\begin{abstract}
College values education has always been an important part of the ideological and political education of college students. With the development of the times and the influence of multiple cultures, the values of college students also show diversity. The growth stage of students in colleges and universities is a critical period for the formation of their values. How to use the correct value education to guide the values of college students is an important issue for colleges and universities. The current rapid economic development and the promotion of globalization have made the links between countries increasingly closer. Chinese and foreign cultural exchanges have become increasingly frequent. Therefore, the problems faced by China in the education of values in universities are also global. In order to break the problem of education in the values of universities in the context of globalization, we must base ourselves on China and have a deep understanding of this issue in an international perspective. From the perspective of culture and cross-cultural perspective, this article based on China's position, using literature research and comparative research methods, analyzes the characteristics of the values education experience in some typical developed countries from different dimensions. Combined with the relevant representative survey data, the existing problems in the education channels and content of Chinese university values education are analyzed, and the innovation of values education should be started from the integration of professional courses, student needs and national reality in order to promote the values of Chinese universities. It hopes to provide theoretical references and practical lessons for the innovation and development of value education in Chinese universities.
\end{abstract}

Keywords: Enlightenment and Reference, Foreign Experience, Values Education

\section{国外经验对中国高校价值观教育实践的启示借鉴研究}

\author{
张文玲 \\ 思想政治教育研究中心, 东北师范大学, 长春市, 中国
}

\section{邮箱}

980707796@qq.com

\begin{abstract}
摘要: 高校价值观教育一直是大学生思想政治教育的重要内容。随着时代发展和多元文化的影响, 高校学生的价值观 也呈现出了多样化的特点。学生在高校的成长阶段是其价值观形成的关键时期, 如何用正确的价值观教育来引导高校 学生价值观, 是当前高校面临的一个重要问题。当前经济飞速发展和全球化的推动, 使得各国之间的联系日益紧密, 中外文化交流日益频繁。因此，中国面临的高校价值观教育问题也具有世界性。要破解全球化背景下的高校价值观教 育问题, 需立足中国, 以国际视野对这一问题进行深刻理解。本文在文化与跨文化的视野下, 立足中国立场, 运用文 献研究和比较研究法, 从不同维度分析部分典型发达国家的高校价值观教育经验特点, 并结合相关具有代表性的调查
\end{abstract}


数据, 分析中国高校价值观教育在教育途径和内容等方面的现存问题, 提出价值观教育创新应从融入专业课程、学生 需求和中国实际三点入手，以期为推动中国高校价值观教育的创新发展提供理论参考和实践借鉴。

关键词：启示借鉴，国外经验，价值观教育

\section{1. 引言}

高校价值观教育一直是中国大学生思想政治教育的 重要内容。中国高校作为培养社会主义事业建设者和接班 人的坚强阵地, 在新时代背景下要立足中国实际, 开展中 外比较研究，在马克思主义理论指导下，充分借鉴吸收对 人类创造有益的理论和实践成果, 推动中国高校价值观教 育的创新发展。由于部分发达国家的高校在通识教育、实 践育人、文化引领等领域有各自独到的经验特点和优势, 值得在结合中国实际的基础上进行吸收借鉴。因此要进一 步完善中国高校价值观教育体系, 眼光不能局限于中国, 在正确对待中国特色的同时还要正确认识世界, 通过考察 部分发达国家的高校价值观教育有益经验, 例如了解掌握 美国、英国、加拿大和日本等国的高校价值观教育内容, 把握其高校价值观教育特点, 并探索出对中国高校价值观 教育具有借鉴的经验和启示, 进而推动中国高校价值观教 育的创新发展。

\section{2. 研究背景和目标}

高校学生作为未来推动社会发展和国家进步的重要人 才, 是未来社会各行业的重要建设者, 更是推动国家和社会 未来发展的中坚力量。因此, 高校学生的价值观教育直接关 系到国家和社会发展的未来。随着经济的快速发展和全球化 时代的到来，高校价值观教育这一问题逐渐成为世界性的问 题, 越来越多的国家认识到高校价值观教育问题的重要性。 并且在研究过程中, 越来越多的中国学者发现了中国高校价 值观教育在教育内容和教育方法等方面的现存问题和挑战。 当前现有研究在高校价值观教育的改革和创新方面取得了 一定的成果，为进一步的研究提供了良好的基础。但是面向 全球化的快速发展, 还有较大的研究空间。一方面现有高校 价值观教育研究需要开阔视野, 在立足中国的前提下, 广泛 开展中外比较研究, 在科学地对比分析中获取高校价值观教 育的有益经验。另一方面, 为进一步推动高校价值观教育的 创新发展, 需要对相关具有代表性的调查研究数据进行深入 分析, 在科学数据和实践案例的支撑下得出具有科学性和准 确性的研究结论, 以便为中国高校价值观教育提供科学正确 的理论指导。总之, 通过对比分析研究部分典型发达国家和 中国高校价值观教育的经验特点, 为高校学生的价值观教育 发展提供正确发展方向, 为中国高校价值观教育理论和实践 创新提供有益借鉴。

\section{3. 研究方法}

研究重点利用文献研究方法和比较研究方法开展研 究, 分析国外部分典型发达国家的高校价值观教育经验,
探寻其中有利于中国高校价值观教育创新发展的经验借 鉴和启示。

第一, 文献研究方法。中国和其他国家的高校价值观 教育研究, 包含内容较多, 能够提供参考和借鉴的资料包 括中国和国外教育领域的众多文献资料, 要深入研究国外 高校价值观教育对中国高校价值观教育创新发展的启示 借鉴，必须从中国和外国部分发达国家的文献资料和相关 调查中深入发掘有价值的资料和内容。因此, 在研究初期 主要针对中国和国外关于高校价值观教育的研究进行了 梳理和综述, 以便准确把握和了解当前中国和其他典型发 达国家的高校价值观教育的研究前沿问题和研究现状, 并 科学预判高校价值观教育的未来发展方向与趋势。同时, 通过文献的阅读和整体的研究方法, 进一步确定了整体研 究的理论框架, 为整体研究提供了科学的逻辑体系。

第二, 比较研究方法。比较研究方法是开展国外高校 价值观教育经验借鉴的重要方法。随着全球化时代的到来, 高校价值观教育需要加强比较研究。在坚持中国立场和明 确中国问题的前提下, 学习借鉴部分国外发达国家高校价 值观教育的有效经验, 促进中国高校价值观教育的创新发 展。研究通过比较研究的方法, 从国外高校价值观教育的 经验特点入手, 对比分析中国和国外在高校价值观教育内 容、方式等方面, 发现中国高校价值观教育的现存问题, 以期为中国高校价值观教育的创新发展提供理论指导和 实践借鉴。同时, 为确保研究结论具有理论价值和实践价 值, 还借助部分学者具有代表性意义的调查数据, 确保研 究论据有数据和实践案例的有效支撑, 保证研究结论的科 学性与准确性。

\section{4. 国外高校价值观教育主要特点}

\section{1. 隐性教育的突出性}

从部分国外发达国家的高校价值观教育中了解到, 这 些国家的价值观教育都有一个共同的特征——隐性教育 突出。由于当今价值观教育对象自身的特点, 所以大多国 外高校价值观教育会以一种柔性化熏染的方式进行价值 观教育, 进而达到价值观教育的目的。例如, 美国政府为 防止美国民众对政府在教育方面制定的国家政策方针有 排斥和反感心理，大多教育方针政策都采取隐性的教育方 式，支持相应的社会组织来淡化民众对于政府干涉教育的 反感心理; 日本则在制服文化中体现爱国主义与集体主义、 在饮食文化中体现实用主义, 以及在各种动漫和电影中体 现价值观教育的相关内容; 加拿大的价值观教育中, 社会 层面的宗教柔性化熏染与学校层面的隐性课程, 这些都突 出了隐性教育。因此, 美国、加拿大和日本的高校价值观 教育形式在一定程度上都采取了隐性教育的方式, 让教育 
对象在潜移默化中接受价值观教育, 甚至达到一种价值观 深入灵魂、日用而不觉的状态。

\section{2. 注重学生综合能力的培养}

从美国、英国、加拿大的价值观教育的研究中发现, 这些发达国家非常注重学生综合能力的培养。以美国和加 拿大为例, 美国的价值观教育中有“价值观教育、道德教 育、公民教育以及品格教育”, 虽然名称不同, 但是都有 一个共同的目标, 就是培养合格、甚至是优秀的公民。特 别是公民教育, 注重培养学生参与公民政治生活的能力, 注重学生未来在社会之中所需能力的培养, 而不仅仅是培 养学生单方面的能力。加拿大的价值观教育, 也注重培养 学生综合能力。以加拿大的安大略省为例, 社会科课程在 $1-6$ 年级为社会科, $7-8$ 年级为历史和地理, $9-12$ 年级 为加拿大与世界研究。社会科、历史、地理以及加拿大与 世界研究等课程一脉相承, 具有共同的教育愿景, 即培养 能够适应社会多样性及负责任的积极公民。帮助学生成为 既见多识广, 又具有批判性精神, 能够自觉珍视包容性社 会, 具有解决问题、有效沟通、明智决策能力的公民。同 时, 英国的价值观教育之中也具有这一特点, 从 SMSC(spiritual,moral,social, cultural development, 简称 $\mathrm{SMSC}$ ）的四个方面来看, 包含精神发展、道德发展、社 会发展以及文化发展这四个方面。每一条的具体内容虽然 不同, 但是能够看出英国学校价值观教育非常注重对学生 的能力培养, 特别是道德发展之中的“区分对错并运用到 生活中的能力”。因此，大部分国外高校价值观教育都十 分注重对学生综合能力的培养, 其高校价值观教育的目标 为培养合格、优秀的公民。在这一过程中，能够积极促进 学生综合能力水平的提升, 进而增强其价值观教育的有效 性。

\section{3. 多层次全方位培养学生}

国外高校价值观教育还具有多层次培养学生的特点。 首先, 以美国的价值观教育为例。美国价值观教育根据学 生自身发展变化的特点采取多层次的教育培养。例如针对 年龄较低一点的学生重点强调道德教育, 而针对年龄较高 的学生则会强调公民教育, 重视其是否有效地参与政治生 活, 重点将其培养成为合格、优秀的社会公民。所以能够 看出美国的整体价值观教育有很多层面的内容, 而且会根 据教育对象的不同年龄阶段来划分层次, 具体问题具体分 析, 针对教育对象的具体实际情况来进行教育, 而不是“一 刀切”。其次, 以加拿大的课程为例。加拿大安大略省的 社会课程教育内容根据两条主线来设计, 一方面是加拿大 遗产和身份认同, 另一方面是加拿大人与环境。例如, $1-6$ 年级的社会科课程主要帮助学生理解“他们是谁”、“从何 而来”，以及“如何能为其赖以生存的社会贡献力量”等问 题。而7-8年级的社会科课程注重历史和地理两个方面, 通过对加拿大历史的学习, 学会欣赏和理解加拿大的文化 遗产与核心价值观，从而形成真正的加拿大国家认同。同 时学习相应的地理知识, 帮助学生了解其赖以生存的家园, 认识自然环境与人类社会发展之间的关系, 从而深度理解 加拿大人的活动及生活方式, 发现加拿大人与其他国家和
民族的不同之处。到了高等教育阶段, 则主要通过通识类 与专业伦理课程开展核心价值观教育。加拿大几乎所有高 校的教学计划中都专门设立了通识教育类课程。由于这类 课程的综合性、理论性和意识形态性较强, 力图将社会主 流价值观的引导与社会道德规范要求融入教育活动中, 因 此这一课程放在高等教育阶段。从美国和加拿大的价值观 教育之中都能看出国外价值观教育多层次全方位培养学 生的特点, 其实不仅是美国与加拿大, 很多国家都会采取 多层次的方式进行价值观教育。既满足了个体的不同发展 需求, 也能在整体的发展之中达到价值观的共识。

\section{5. 中国高校价值观教育的现存问题}

当前中国高校价值观教育已取得较好进展, 但是在教 育途径、教育内容和教师能力建设等方面还存在一些问题, 导致价值观教育过程中无法形成教育合力, 进而影响高校 价值观教育的整体性效果。

\section{1. 教育途径过于单一}

高校价值观教育的教育途径包含课堂教学、课外活动 和校园文化三种基本途径, 但是当前中国高校价值观教育 过程中, 出现了价值观教育过分注重课堂教学的现象。例 如, 2016年度中国大学生思想政治教育发展报告中显示, 被调查的 $50.4 \%$ 的大学生认为当前思想政治教育理论课的 教育效果“一般”, 还有 $10 \%$ 左右的学生认为当前思想政治 教育理论课在课程设置、教学内容和教学途径方法等方面 还有待提升。参与调查的学生希望能够增加一些不同的教 育途径, 例如增加一些实践类教学, 而不是只采取“课堂 灌输”的教育途径。因为单纯的课堂灌输式教学难以激发 学生对价值观教育的学习兴趣, 导致高校价值观教育效果 不佳。虽然课堂教学是高校价值观教育的主渠道, 必须用 好课堂教学, 但是过分强调课堂教学, 忽略其他教育途径 就会违背价值观教育的传播特点和价值要求, 会影响高校 价值观教育的整体教育效果。

\section{2. 教育内容缺乏针对性}

长期以来, 中国高校价值观教育没有较为明显的层次 划分和针对性，特别是没有以思想政治工作规律、教书育 人规律、学生成长规律这三大规律为出发点进行核心价值 观教育, 进而导致中国高校价值观教育的实效性较差。 2016年度中国大学生思想政治教育发展报告中显示, 当前 不同年龄阶段、不同学科的大学生群体在价值观方面呈现 出显著差异。正如表1所示, 理工类学生与人文、社科类 学生相比, 其核心价值观理解程度偏低, 并且整体上硕士 生对于核心价值观的理解程度高于本科生的理解程度。可 见, 当前高校学生由于学习阶段和学科的不同, 因此在核 心价值观的理解程度上还是存在着较大差异。并且被调查 的学生还表示价值观教育内容改进的重点应在于“密切与 现实生活的联系” (69.0\%) 和“积极回应社会热点问题” (60.4\%)。从上述数据中可以分析提出, 当前高校价值 观教育取得了一定的成效, 但是没有特别突出强调学生的 群体差异, 导致不同学生群体由于学习阶段和学科的差异, 
在核心价值观理解方面呈现出较大差异, 整体上影响了价 值观的教育效果。同时, 学生在价值观教育内容方面着重 强调“密切与现实生活的联系”和“积极回应社会热点问题” 的内容, 可见以往的教育内容中在一定程度上忽视了这两 点内容, 这一现象的产生导致其教育内容缺乏针对性和吸 引力, 学生难以从中满足自身的发展需求, 也无法在教育 过程中提升自己的知识水平和能力, 导致中国高校价值观 教育缺乏实效性和针对性。

表1 2016年度中国大学生核心价值观理解程度差异 (不同学习阶段、不 同学科）。

\begin{tabular}{lllll}
\hline \multirow{2}{*}{ 学习阶段 } & \multirow{3}{*}{$\begin{array}{l}\text { 对社会主义核心价值观的理解程度 } \\
\text { (单位: \% }\end{array}$} \\
\cline { 3 - 5 } & & 高 & 中 & 低 \\
\hline \multirow{3}{*}{ 本科 } & 人文 & 68.6 & 26.9 & 4.5 \\
& 社会 & 61.4 & 31.3 & 7.3 \\
& 理工 & 56.3 & 33.5 & 10.2 \\
\multirow{4}{*}{ 人硕士 } & 人文 & 74.2 & 24.7 & 1.1 \\
& 社会 & 75.6 & 21.3 & 3.2 \\
\multirow{4}{*}{ 博士 } & 理工 & 67.4 & 23.7 & 8.9 \\
& 人文 & 67.9 & 21.4 & 10.7 \\
& 社会 & 75.4 & 21.5 & 3.1 \\
& 理工 & 67.8 & 25.5 & 6.7 \\
\hline \multirow{2}{*}{} & & & &
\end{tabular}

\section{3. 教育者综合教学能力不足}

中国高校价值观教育体系中, 存在着部分教育者教学 能力不足的现象。根据2016年度大学生思想政治教育状况 的调查数据, 结果显示大学生针对教育者的教学方法的建 议集中在“加强师生互动交流 (18.3\%) ”、“重视开展实践 教学 $(26.3 \%)$ ）、“重视网络、多媒体等教学手段的运用 $(9.5 \%)$ )、“增强教学趣味性 (31.3\%) ”、“强化案例教 学 (14.6\%) ”这五个方面。从以上数据和学生提出的建议 来看, 高校教师的综合教学能力不足, 水平有待提升。当 前很多高校教师在进行价值观教育和教学时, 其讲授和阐 述过于理论化和学术化, 存在教育内容陈旧、教学感染力 不强等现象, 降低了高校价值观教育吸引力、忽视了价值 观教育的实践教学以及与学生的互动交流, 进而影响了价 值观的教育效果, 因此目前中国高校教师所采用的教学方 法有待改进和提升。同时, 根据学生提出的教学方法改进 建议不难看出, 学生认为当前教学方式在以上五个方面需 要提升, 在一定程度上反映了教育者在这些方面的欠缺。 因此当前中国高校价值观教育者存在综合教学能力不足 的问题, 其教育理念和综合教学水平亟待提升。

\section{6. 国外高校价值观教育对中国高校价值观教育 的启示}

\section{1. 价值观教育应融入专业课程中}

从国外高校价值观教育的特点中了解到, 国外很多高 校都会通过一些通识类和专业伦理课程展开核心价值观 教育, 将社会主流的价值观引导与社会道德规范要求融入 教育活动之中, 既具有隐性教育的特点, 又达到了核心价 值观教育的目的。例如有学者针对孔子学院师生和来华人 员进行调查, 在价值观教育效果的调查中发现, 被调查者
对于所属国家的价值观教育课程总体评价较好，有 $36.7 \%$ 的人认为很有效果, 仅有 $6.1 \%$ 的人认为效果不佳。并且, 被调查者中有 $49.5 \%$ 的人认为价值观教育渗透于课程中的 教育效果明显, 有 $28.4 \%$ 的人认为技术类课程中开展的价 值观教育效果明显。因此中国高校价值观教育一方面应当 将核心价值观教育内容融入专业课程之中, 让学生在学习 专业课程的同时, 潜移默化地受到价值观教育的影响。同 时也要在校园文化中融入价值观教育内容, 根据国外高校 价值观教育状况调查显示, $58.6 \%$ 的被调查者认为校园文 化和校园环境中所蕴含的价值观教育成效显著。因此, 中 国高校价值观教育还要将价值观教育内容融入校园文化 和环境中，形成教育合力，提升中国高校价值观教育的实 效性。另一方面, 依据现有思想政治理论课程, 明确课程 的职责与培养目标。以日本新的《学习指导要领》为例, 要领之中会根据每一条内容的核心思想提炼每一项内容 的关键词。便于学生理解的同时, 也有助于教师对道德教 育教学的把握。更重要的是帮助教师与学生确定了道德教 育的关键词, 明确了道德教育的目标与方向。借助这一方 式不仅能够让学生们接受价值观教育、明确自己的任务, 同时有利于高校价值观教育的完善与发展。

\section{2. 以学生成长成才的需求为出发点, 加强学生道德判 断能力}

国外高校价值观教育之中有一个明显特征——分阶 段、分层次培养学生。以加拿大的高校教育课程为例, 他 们将社会科课程划分为三个阶段, 由浅到深, 依据年级的 高低和各年级学生的特点来划分教育的内容与形式, 并根 据学生成长为合格、优秀公民的实际要求来划分价值观教 育的层次, 既能保证各年级学生价值观教育的质量, 又能 保证学生在未来的发展过程中将所学的相关内容学以致 用, 为国家和社会的发展做出相应的贡献。除此之外, 依 据学生的成长成才需要进行价值观教育的还有美国的众 多高校。有学者曾对美国的阿尔维诺学院、加州大学蒙特 瑞湾分校、圣凯瑟琳学院、杜克大学等十二所美国高校的 道德和公民发展进行调研, 这些学校虽然类别有所不同, 但是在价值观教育方面都会坚持从学生的能力和需求出 发, 确保价值观教育能够推动学生的发展, 这一做法得到 了大量的关于学生进步发展的反馈。并且根据国外高校价 值观教育状况的调查数据显示, 参与调查的群体中有 $40.5 \%$ 的人认为价值观教育采取“价值澄清理论”进行教学 有利于教育效果的提升。所谓价值澄清就是根据学生个人 的道德、品德的发展阶段和实际发展需要, 借助多种有效 的途径和方式, 让学生在轻松愉快的环境中获得成效显著 的价值观教育, 并收获积极的人生态度, 从而树立正确的 世界观、人生观和价值观。同时, 要加强对学生道德判断 能力的培养。当今高校运用的价值观教育方法, 大多采用 理论灌输的方法, 直接向学生灌输正确的价值观, 让学生 无条件的接受正确的结论。尽管这种方法能够让学生快速 地了解价值观的正误, 但是却妨碍了学生独立思考和判断, 学生无法真正掌握道德判断的方法。因此, 在满足学生成 长成才的同时还需要加强对学生道德判断能力的培养。这 种能力的培养不能简单地通过课堂上的讨论法、案例法等 
教学方法进行加强, 需要在符合现实生活的情境中进行。 教师可以创设一些教育情境, 让学生在各种各样的教育情 境中, 通过思考和讨论, 对现实生活中存在的种种矛盾进 行分析和权衡之后, 阐明自身的立场和观点, 从而提升学 生自身的道德判断能力。

\section{3. 依据中国实际, 批判性地借鉴、吸收国外价值观教 育经验}

在借鉴国外价值观教育时, 最重要的是比较借鉴的态 度与方法。无论国外高校价值观教育有怎样的优势与特点, 都需要结合中国高校价值观教育的现状和特点, 进行批判 性地借鉴, 有选择性的吸收。美国著名教育哲学家、关怀 伦理创始人内尔・诺丁斯（Nel Noddings）在《21世纪的教 育与民主》中指出, “教育领域内根本不存在一种所谓“包 治百病的灵丹妙药”, 尽管“在教育史上曾经涌现出许多卓 越的想法, 但许多已经消解在泛化的滥用之中了。”同时, 根据国外高校价值观教育状况的调查数据显示, $70.8 \%$ 的 被试者认为在全球化和价值多元化的今天, 每个国家应该 有自己的价值观教育发展模式和道路, 主张多元价值观的 同时, 也要明确自身的价值观和价值观教育。中国的历史 发展也证明, 全盘吸收外来文化的结果就是迷失自己, 只 有根据中国国情发展和实际需要进行批判性的借鉴和吸 收, 才能获得真正的发展。由此可见, 要真正提升中国高 校价值观教育, 比较借鉴只是提升的手段和方法, 而不是 最终的教育目的。因此, 在借鉴吸收国外高校价值观教育 经验时应注意以下两点。首先, 在借鉴、比较时要保持自 信, 特别是对中国高校价值观教育的自信。在进行借鉴时, 需要树立自身的价值立场, 不能盲目地借鉴国外价值观教 育的经验。在这一过程中, 就需要坚定中国特色社会主义 发展道路, 正确认识中国特色和国际比较。其次, 结合中 国实际进行比较、借鉴。中国之所以借鉴国外高校价值观 教育的经验, 是为了解决中国高校价值观教育面临的问题 与困境, 这些问题和困境是立足于中国国情与实际的。所 以比较借鉴时除了取长补短, 更重要的是为中国所用。因 此, 要站在中国立场上, 秉持中国意识, 解决中国问题, 有选择性地吸收和借鉴国外价值观教育经验, 而不是盲目 的借鉴和自信。

\section{7. 结论}

总之, 在比较借鉴国外高校价值观教育有益经验的过 程中, 遵循高校价值观教育的发展规律与学生成长成才规 律, 站在中国立场上, 批判性地借鉴吸收国外高校价值观 教育的经验, 不断创新和完善中国高校价值观教育的内容
与体系, 并增强中国进行国际比较的自信。使中国在全球 化国际竞争中, 进一步完善和发展中国高校价值观教育的 内容与体系, 让中国的价值观教育为人类贡献中国价值、 中国智慧、中国经验和中国方案, 最终达到“各美其美, 美美与共”的价值理想。

\section{参考文献}

[1] Colby, A., Ehrlich, T., Beaumont, E. \& Stephens, J. (2010). Educating Citizens: Preparing America's Undergraduates for Lives of Moral and Civic Responsibility [M]. Wiley: Jossey-Bass.

[2] 杨晓慧.思想政治教育前沿译丛 [M]. 北京: 人民出版社, 2015。

[3] 柳海民. 教育原理[M]. 长春: 东北师范大学出版社, 2006: $126-137$ 。

[4] 檀传宝. 学校道德教育原理[M]. 北京: 教育科学出版社, 2015。

[5] 沈壮海,王迎迎.2016年度大学生思想政治教育状况调查分 析一一基于全国35所高校的调查[J].中国高等教育,2017, 587(11):45-50。

[6] 沈壮海,肖洋.2016年度大学生思想政治状况调查分析 [J].思 想理论教育导刊,2017，217(01):108-113。

[7] 王汉林,刘诚,戴玉琴,张晊.国外高校价值观教育状况及启示 一一对国外及来华人士的调查报告[J].扬州大学学报(高教 研究版),2013,17(05):7-10。

[8] 杨威. 国外价值观教育研究:目标、内容与方法[J].思想理论 教育, 2017, 463(10): 10-16。

[9] 杨威. 国外价值观教育的当代复兴及研究现状 $[J]$. 教学与研 究, 2017, 51(9): 103-112。

[10] 李欣怡,任成孝,高金金. 国外学生核心价值观教育研究及其 当代启示一一基于马克思主义核心价值观的思考 [J]. 教育 理论与实践, 2015, 35(13):42-45。

[11] 刘洪波.高校社会主义核心价值观教育探析一一基于美国 核心价值观教育的经验 [J]. 思想政治教育研究, 2016,32(04):9-12。

[12] 朱飞. 高校思想政治工作须注重方法创新——以价值观教 育为视角 [J].思想教育研究, 2016, 264(07):101-103+107。

[13] 夏海燕. 反思与建构:开放德育视域下的高校价值观教育 [J]. 南京师大学报(社会科学版), 2017, 209(01):85-91。 